\title{
Star polymers
}

It is our great pleasure to present a collection of papers devoted to theoretical, numerical, and experimental studies in the field of star polymers. Since its introduction in the early 80-ies, this field has attracted increasing interest and has become an important part of contemporary polymer physics. While research papers in this field appear regularly in different physical and chemical journals, the present collection is an attempt to join together the studies of star polymers showing the variety of techniques that have been developed to establish a sound understanding of their properties.

To give an idea about the subjects covered by the papers published in this collection, let us group them according to their main focus. Dynamical properties of star polymers and of the related systems are studied in the papers by F.Ganazzoli, and by M.L.Mansfield and J.F.Douglas, as well as in experimental papers by D.Vlassopulos, T.Pakula, and J.Roovers, and by C.Branca, S.Magazù, and F.Migliardo. Structure and thermodynamics of star-polymer-like molecule fluids is a subject of the articles by Yu.Kalyuzhnyi and M.Holovko and by Yu.Duda and A.Trokhymchuk. Phase diagrams of the systems containing star polymers are analyzed in the papers by C.N.Likos and H.M.Harreis and by J.Dzubiella and A.Jusufi. Questions of confinement are examined by P.D.Guirati and by P.Romiszowski. The scaling properties of star polymers are reviewed by K.Ohno, by L.Lue and S.B.Kiselev, and by C. von Ferber and Yu.Holovatch.

All the above papers appear in the first two issues of volume 5 of "Condensed Matter Physics" (2002). The total number of pages of the papers we have received in response to our invitation exceeds the maximal size of a single journal issue. While we are really glad about this very positive response, we need to include several contributions in the second issue of volume 5. These are the articles "Star-polymercolloid mixtures" by J.Dzubiella and A.Jusufi, "Numerical path-integration calculation of transport properties of star polymers and DLA aggregates" by M.L.Mansfield and J.F.Douglas, "Polymer mean spherical approximation for the fluid of flexible hard-sphere Yukawa star molecules" by Yu.Kalyuzhnyi and M.Holovko, "Star polymer/water solutions: new experimental findings" by C.Branca, S.Magazù, and F.Migliardo, "Towards a simplified approach to the modelling of the star-like molecule fluids" by Yu.Duda and A.Trokhymchuk.

We express our appreciation to all the authors for their commitment and gratitude for their cooperation during the editorial stages. It is also a pleasure to thank Viktoria Blavats'ka and Maxym Dudka for their technical assistance.

Freiburg and Lviv, February 2002,

Christian von Ferber, Yurij Holovatch 
